\title{
Bioanalysis
}

\section{Recommendations for classification of commercial LBA kits for biomarkers in drug development from the GCC for bioanalysis}

Over the last decade, the use of biomarker data has become integral to drug development. Biomarkers are not only utilized for internal decision-making by sponsors; they are increasingly utilized to make critical decisions for drug safety and efficacy. As the regulatory agencies are routinely making decisions based on biomarker data, there has been significant scrutiny on the validation of biomarker methods. Contract research organizations regularly use commercially available immunoassay kits to validate biomarker methods. However, adaptation of such kits in a regulated environment presents significant challenges and was one of the key topics discussed during the 12th Global Contract Research Organization Council for Bioanalysis (GCC) meeting. This White Paper reports the GCC members' opinion on the challenges facing the industry and the GCC recommendations on the classification of commercial kits that can be a win-win for commercial kit vendors and end users.

First draft submitted: 22 February 2019; Accepted for publication: 13 March 2019; Published online: 17 April 2019

Keywords: BAV • bioanalysis $\bullet$ biomarkers $\bullet$ commercial kits $\bullet$ CRO $\bullet$ immunoassay $\bullet$ GCC $\bullet$ LBA

The Global Contract Research Organization Council (GCC) for Bioanalysis was formed in 2010 to bring together many senior-level contract research organization (CRO) representatives to openly discuss bioanalysis and the regulatory challenges, pertinent to the outsourcing industry. CROs work with many different sponsors, vendors and regulatory agencies, which results in unique and comprehensive perspectives on scientific approaches in relation to regulatory requirements. Since the formation of this international consortium at the 1st GCC Closed Forum held on 14 September 2010 in Montreal, Canada [1], there have been meetings in North America and Europe [2-9]. Furthermore, GCC has published its official recommendations in White Papers [10-15], which were well received within the regulated bioanalytical arena, including regulatory agencies. In an effort to accommodate the schedules of the CRO representatives, GCC meetings will continue to be tied to major conferences where attendance by member companies is anticipated. More information on the GCC unique structure can be found in the publication titled 'Formation of a GCC for Bioanalysis' [1].

\section{Introduction}

The adaptation of commercial test kits in a regulated environment was one of the key topics discussed during the 12th GCC Closed Forum [Briscoe et al. 12th GCC Closed Forum, In preparation]. The interest in using commercially available, research use only (RUO) immunoassay kits has steadily grown with the demand for biomarker analysis in drug development. However, commercial kits typically must be adapted by bioanalytical laboratories before validation of the assay because the kits are usually meant for drug discovery or clinical diagnostics as opposed to drug development. There is an unmet need for 'well-defined' commercial kits that can be utilized in a drug development setting [16]. However, there are no standardized qualification criteria, and each kit manufacturer has their own specific requirements for kit characterization and documentation. For example, kits are often supplied with materials for testing in buffer

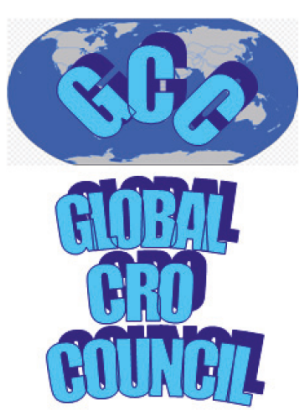

Rafiq Islam ${ }^{1}$, Sumit Kar ${ }^{1}$, Hanna Ritzén ${ }^{2}$ et al.

*Author for correspondence: Global CRO

Council for Bioanalysis;

wei@global-cro-council.org

Disclosure: Due to the equality principles of Global CRO Council for Bioanalysis, the authors are presented in alphabetical order of member company name, with the exception of the first four authors who provided major contributions to this paper.

The full list of author names and affiliations can be found at the end of the article 
where the vendor does not evaluate matrix interference. Other challenges include a lack of standard definition of sensitivity, undetermined lot-to-lot variability, and lack of identification and documentation for critical reagents and reference material certificate of analysis (CoA or RoA). As a result, the required assay quality may not be achieved in a cost-effective or timely manner.

There have been previous calls for commercial kits that are characterized specifically for drug development use in a regulated environment. Bowsher et al. called for 'pharmaceutical grade kits' that would comply with clear criteria and documentation that are suitable for use in a regulated environment [17]. Islam et al. proposed a three-tiered approach to 'drug-development kits' with clearly defined quality attributes that can be adapted to perform fit-forpurpose (FFP) analytical validation in a regulated environment [18]. While some commercial kit manufacturers have begun to classify their kits according to higher levels of characterization (e.g., 'V-plex' from MesoScaleDiscovery, 'regulated kits' from Somru Bioscience, Inc.), there has not been an industry-wide effort between manufacturers and the end users to improve the overall quality of kits for use in drug development. As GCC members are one of the key end users of commercial kits, the community felt it necessary to address this topic. We believe that a mutual lack of communication has helped foster a protracted misunderstanding between manufacturers of the kits and end users in regulated bioanalytical laboratories. The GCC believes that the specific criteria highlighted in this White Paper will provide an attainable framework for kits suited for bioanalysis in drug development.

It is worth mentioning that GCC members have been experiencing increased regulatory scrutiny on biomarker assay validation (BAV) using commercial kits. This is also evidenced by the most recent US FDA bioanalytical method validation (BMV) guidance (May 2018) which requires stringent bioanalytical requirements for biomarkers, and includes guidance for adapting commercial diagnostic kits, and details the documentation necessary for validating assays, including CoA for critical reagents [19].

Thus, this White Paper highlights the need the bioanalytical community has for commercial kits able to be used for regulated drug development. The GCC provides a framework for the creation of drug development kits. Importantly, GCC recommendations are focused on single-plex immunoassay kits that measure biomarkers. The proposals exclude multiplex immunoassay kits, flow cytometry kits or kits used for pharmacokinetic assays and US FDA cleared kits for diagnostic purposes. The GCC does not intend for these recommendations to be a strict regulation for vendors but rather the start of a dialog between the customers of these kits and manufacturers, to be able to provide more reliable, cheaper, and higher quality drug products for patients in need.

\section{Biomarker assay validation}

Biomarker assays must undergo a 'FFP' validation based on the context of use (COU) of the biomarker [20-22]. It is well understood that a 'FFP' validation is required for biomarker assays during drug development. The extent of the validation is dependent on the role of the biomarker in the safety and efficacy of the new drug product. In general, BAV should address general validation parameters that pertain to the characterization of the assay including accuracy, precision, sensitivity, selectivity, dilutional linearity, parallelism, range, reproducibility and stability. BMV and validation parameters have been extensively discussed in previous publications and are outside of the scope of this White Paper [14,16,23-28]. In this publication, we focus on the classification of commercial biomarker kits and how this proposed classification aligns with the extent of kit validation. This classification provides bioanalytical laboratories a standardized expectation on kit performance before purchase and subsequent validation.

In general, there is an agreement within the global bioanalytical community that three levels of validation are needed and utilized [21,23]. The three levels of validation allow biomarkers to be validated for different COU, which is 'a statement that fully and clearly describes the way the medical product tool is to be used and the medical product development related purpose of use' [29]. The proposed two-level kit classification schemes in the GCC recommendations section follow the spirit of this three-tier assay validation approach. The GCC believes the purpose of classifying commercial kits is to align with the effort needed for each validation tier and provide bioanalytical laboratories a standardized expectation of the contents of commercial kits before validation to minimize time, cost and regulatory hurdles. The GCC understands that the classification by the vendor in no way removes responsibility from the bioanalytical laboratories who must validate immunoassay kits to the necessary purpose no matter the classification of the kit by the vendor. 
Table 1. GCC survey on commercial kit classification.

\begin{tabular}{|c|c|}
\hline Question & Answer \\
\hline $\begin{array}{l}\text { What type of commercial kits do you use for bioanalysis in drug-development } \\
\text { projects? }\end{array}$ & $\begin{array}{l}\text { - Research use only: } 45 \% \\
\text { - In vitro diagnostic: } 17 \% \\
\text { - Conformité Européenne: } 15 \% \\
\text { - CLIA laboratory developed test: } 3 \%\end{array}$ \\
\hline What is the intended use of the biomarker data you generate? & $\begin{array}{l}\text { - Exploratory: } 38 \% \\
\text { - Internal decision-making: } 31 \% \\
\text { - Data supporting regulatory submission: } 29 \% \\
\text { - Other (PK/PD): } 2 \%\end{array}$ \\
\hline $\begin{array}{l}\text { Which features do you most like about commercial kits? (rank 1-6: } 1=\text { most liked; } \\
6=\text { least liked) }\end{array}$ & $\begin{array}{l}\text { 1. Convenience } \\
\text { 2. Specificity and crossreactivity data } \\
\text { 3. Performance data } \\
\text { 4. Precoated plate } \\
\text { 5. Cost-effective } \\
\text { 6. Other (protocol provided, IVD/FDA cleared, reagents can be optimized for disease } \\
\text { states) }\end{array}$ \\
\hline $\begin{array}{l}\text { What features do you desire in commercial kits? (rank 1-9,1 = most desired; } 9= \\
\text { least desired) }\end{array}$ & $\begin{array}{l}\text { 1. Well-characterized reference material } \\
\text { 2. Lot-to-lot variability data } \\
\text { 3. Parallelism data } \\
\text { 4. Critical reagent characterization } \\
\text { 5. }>6 \text { STDs } \\
\text { 6. >3 QCs } \\
\text { 7. LLOQ determined in addition to LOD } \\
\text { 8. Prompt technical support } \\
\text { 9. Other (stability data, standard matrix data, safety data) }\end{array}$ \\
\hline
\end{tabular}

\section{Summary of survey data}

A survey was circulated to GCC membership to determine the different approaches to using commercial kits. The goal was to form a consensus on the needs and appropriate classification of kits for drug development. The results of this survey are presented in Table 1.

From the survey, the most common commercial kits used for biomarkers by GCC members are research use only (RUO) kits. Therefore, GCC recommendations for drug development kits characterized for bioanalysis focuses on improving the characterization of RUO kits. In vitro diagnostic, Conformité Européenne and laboratory developed tests, while also adapted and used by members, are not included in this classification approach because they are already classified by regulatory bodies such as the US FDA, EMA, Clinical \& Laboratory Standards Institute and Clinical Laboratory Improvement Amendments.

The survey also showed that the intended use of biomarker studies that utilize commercial kits spans an almost even mix of exploratory, internal decision making and data supporting a regulatory submission. This response supports the need for commercial kits that are better classified and aligned with the three different COU categories $[21,23]$.

Most importantly, GCC members were asked to list features they currently like and features they desire from commercial kits. The top three most appreciated commercial kit features are convenience, specificity/crossreactivity data and performance data. The most desired features for kits are a better characterization of reference material and critical reagents as well as the inclusion of both lot-to-lot variability and parallelism data. As such, the addition of consistent documentation of performance, parallelism, lot variability and critical reagent data is the pivotal intent of the GCC's recommendations for commercial kits to improve the convenience and value to the regulated bioanalysis user.

\section{GCC recommendations}

GCC proposes the formation of 'Drug Development Kits' (DDK), which are designed to address the needs of biomarker work in drug development. Discussion at the 12th GCC Closed Forum [Briscoe et al. 12th GCC Closed Forum, In preparation] addressed the format of the classifications and whether multiple tiers of kits are needed to be consistent with the three-tier biomarker validation framework. While three tiers of drug-development kits would best align with the validation framework for assays and the effort necessary to adapt a kit for each tier, this may cause unnecessary complexity for vendors with multiple stock keeping units for each biomarker. The consensus was 
Table 2. Classification of parameters for the formation of drug-development kits.

\begin{tabular}{|c|c|c|}
\hline Parameter & Drug-development kit & Standard RUO kit \\
\hline Intended use & $\begin{array}{l}\text { Regulatory submission/full validation/partial } \\
\text { validation }\end{array}$ & Exploratory validation/partial validation \\
\hline Reference material & $\begin{array}{l}\text { Must be traceable to internationally recognized } \\
\text { reference (i.e., WHO, NIBSC and NIST) or well } \\
\text { characterized with a CoA as per Table } 3 \text { to match } \\
\text { endogenous biomarker }\end{array}$ & Must be characterized as per Table 3 \\
\hline Calibrator diluent & $\begin{array}{l}\text { Matrix-based preferred; when surrogate matrix is } \\
\text { used that matrix should be defined, and absence of } \\
\text { matrix effect should be demonstrated. If surrogate } \\
\text { matrix is used, the rationale for its use should be } \\
\text { explained along with contents of matrix }\end{array}$ & Surrogate matrix \\
\hline Number of nonzero calibrators & $\geq 6$ & $\geq 6$ \\
\hline Parallelism & Should be tested and documented & Optional \\
\hline Selectivity/matrix effect & $\begin{array}{l}\text { Matrix effect experiments should be performed using } \\
\text { the intended matrix }\end{array}$ & Optional \\
\hline Specificity/crossreactivity & $\begin{array}{l}\text { Crossreactivity to structurally similar analytes or } \\
\text { analytes in same biological pathway should be } \\
\text { performed }\end{array}$ & Optional \\
\hline Precision and accuracy & $\begin{array}{l}\text { Must be performed with QCs prepared from screen } \\
\text { matrix samples and at least one endogenous QC } \\
\text { including LLOQ and ULOQ }\end{array}$ & $\begin{array}{l}\text { Surrogate matrix is acceptable; the endogenous matrix } \\
\text { is recommended. Sensitivity accounts for the minimum } \\
\text { required dilution (reportable range) }\end{array}$ \\
\hline Quality control samples & $\begin{array}{l}\text { Endogenous QCs and additional QCs of analyte spiked } \\
\text { in the intended matrix with known concentrations. Or } \\
\text { provide a high concentration stock of analyte for QC } \\
\text { preparation by the user }\end{array}$ & Surrogate matrix QCs acceptable \\
\hline Lot-to-lot variability & $\begin{array}{l}\text { Should be tested and controlled or documented with } \\
\text { bridging data (including changes in critical reagents) }\end{array}$ & Optional \\
\hline Limits of quantitation vs limit of detection & $\begin{array}{l}\text { Limit of quantitation should be defined, and it should } \\
\text { be specified if it is in surrogate matrix or endogenous } \\
\text { matrix (endogenous matrix recommended) }\end{array}$ & Limit of detection is sufficient \\
\hline Documentation & $\begin{array}{l}\text { Should identify and include CoA for reference } \\
\text { material, critical reagents and kit performance data } \\
\text { detailing experimental parameters and results. Should } \\
\text { include data of the above stated parameters }\end{array}$ & $\begin{array}{l}\text { Documentation should include kit performance data } \\
\text { summarizing experimental parameters and results }\end{array}$ \\
\hline
\end{tabular}

reached that there is sufficient need for kit manufacturers to create a level of kits called DDKs that are validated by the vendor beyond their existing RUO kits.

A proposed framework for the validation tests and documentation for DDKs and RUO kits are shown in Table 2. With this model, DDKs are intentioned for full biomarker method validations in support of data submission to a regulatory agency and partial validations for internal decisions using biomarkers (Tier II and III COUs). The RUO kits are best suited for exploratory COU. It is important to note that this does not preclude a RUO kit from being used for higher levels of validation (and vice versa) but would require appropriate adaptation for the FFP validation/COU. The GCC also recognizes that vendors, who are already developing kits with additional validation, are not likely willing to change their trademarked branding. However, the tests and documentation standards presented here can set a bar for those existing kit classifications for a consistent and transparent level of quality across the industry. In the next section, we detail the specific components a drug development kit should contain to be considered appropriate for bioanalytical use.

\section{Key components of drug-development kits}

Based on the survey responses, the GCC recommendations focus on five key components that DDKs must contain. These are well-characterized reference material and critical reagents, lot-to-lot variability data, parallelism data and appropriate QCs.

\section{Reference material}

Reference materials for biomarkers are challenging because of the difficulty of determining the similarity of the calibrator materials to the endogenous analytes [24,30]. Tests such as parallelism discussed below, help assess the 
Table 3. GC GCC recommended characterization requirements for reference material and critical reagents in commercial kits.

\begin{tabular}{|c|c|c|}
\hline Attributes & Drug-development kit & Standard RUO kit \\
\hline \multicolumn{3}{|l|}{ Reference material } \\
\hline Molecular weight and species & Yes & Yes \\
\hline Concentration & Yes & Yes \\
\hline Potency & Yes & Optional \\
\hline Purity & Yes & Yes \\
\hline Aggregation level & Yes & Optional \\
\hline Lot-to-lot bridging & Yes & Optional \\
\hline $\begin{array}{l}\text { Traceable to internationally recognized (i.e., NIBSC, WHO, NIST, etc.) reference } \\
\text { materials }\end{array}$ & Yes & Optional \\
\hline Parallelism testing using endogenous biomarker, when possible & Yes & Optional \\
\hline \multicolumn{3}{|l|}{ Critical reagents (i.e., coating and detection antibodies) } \\
\hline Concentration & Yes & Optional \\
\hline Binding activity & Yes & Optional \\
\hline Formulation buffer specified & Yes & Optional \\
\hline The identity of antibody including species, isotype & Yes & Optional \\
\hline Functional assay & Yes & Optional \\
\hline Stability & Yes & Optional \\
\hline Crossreactivity to structurally similar molecules & Yes & Optional \\
\hline Conjugate incorporation ratio & Yes & Optional \\
\hline
\end{tabular}

similarity of reference materials to endogenous analytes. Also, use of international reference standards such as those from WHO, NIBSC, USP and NIST establishes confidence in the consistency and similarity of reference standards. Kit manufacturers may bridge their reference materials to these international standards. However, only a limited number of international reference standards have been established. When unavailable, assignment of reference values should be documented through characterization of purity, potency and concentration of the material by the end user and by the vendor for DDKs (Table 3). The US FDA BMV Guidance also stipulates [19] the sponsor should provide the CoA with the source, lot number and expiration date for commercially available reference standards. Following bioanalytical assay guidelines, the reference material would then be spiked into control matrix to produce a calibration curve with at least six calibrator points (though eight are preferred to allow masking for failed standards) including the LLOQ and ULOQ.

\section{Critical reagents}

Critical assay reagents are essential components of LBA utilized throughout the process of drug discovery, development and postmarketing monitoring $[16,24,31]$. The characteristics of these reagents can have a significant impact on assay reliability and reproducibility. The US FDA BMV guidelines describe critical reagents as reference standards, antibodies, labeled analytes and matrices, and goes on to stipulate these reagents should have documented identity, purity and stability. For kit-based assays, these properties are difficult to determine if not provided by the kit vendor.

Discussion at the 12th GCC Closed Forum [Briscoe et al. 12th GCC Closed Forum, In preparation] evaluated which reagents should be categorized as critical reagents because even reagents such as buffers can have a critical impact on assay performance and lot-to-lot variability. It was recommended that a risk-based approach should be used when identifying critical reagents as they will vary by assay and COU. However, at minimum, the critical reagents listed by the US FDA are considered critical [19]. In DDKs, the concentration, binding activity, formulation buffer, species identity, stability and conjugate incorporation ratio should be defined in the CoA. The characterization recommended by the GCC for critical reagents is described in Table 3.

\section{Lot-to-lot data}

Variability of kit performance across lots and changes in critical reagents significantly impedes the adaptation of commercial kits, especially for long-term clinical studies. While changes in critical reagents may individually meet 
a vendor's release criteria, when combined with other kit components, including matrix, assay performance can be significantly impacted. For DDKs, this can be partially controlled by the manufacturer by implementing rigorous manufacturing processes, automation, uniformity testing, and quality control. Manufacturers can also implement procedures to monitor consistency of performance across lot changes. For example, accuracy and precision of endogenous QCs can monitor assay performance. These QCs can also be used to perform trend analysis over time (e.g., Westgard rules, Shewhart or Levey-Jennings control plots) [32]. Performance differences between lots can then be investigated and sufficiently mitigated. Alternately, lot-dependent performance changes may call for the use of an empirical correction factor to be applied to experimental data [33,34]. Regardless of the method used, providing this data as part of a DDK allows the validating laboratory to determine whether the kit fits the COU and length of the study. The US FDA BMV Guidance [19] requires sponsors to evaluate the performance of QCs and standard curves, binding activity and crossreactivities with changes in critical reagent lots and assistance from manufacturers in this regard can only help the uptake of commercial kits.

\section{Parallelism data}

Parallelism is a significant performance characteristic of biomarker assays that demonstrate that the endogenous sample response curve is parallel to the calibration curve. It is also used to detect potential matrix effects and interactions between critical reagents in an assay. However, parallelism is rarely evaluated and documented for commercial kits. Previous White Papers and publications have discussed different approaches for performing parallelism [35-39]. However, the one recommended by the US FDA BMV Guidance is to serially dilute high concentration endogenous samples and compare them with a dilution of the reference standard. The GCC does not intend to dictate how parallelism should be performed and analyzed due to the difficulty in obtaining high concentration analytes for many biomarkers and the complexity of the interpretation of the data. However, some form of parallelism should be performed with the results documented in DDKs for the validating lab to determine if the reference material is suitable for the 'FFP' validation.

\section{Quality controls}

QC samples are typically included in kits to determine assay performance during sample analysis. However, the number and type of QCs included in a commercial kit are highly variable. Often, QCs are provided with an acceptable range of concentrations based on readings from the kit itself and not based on a percentage of the nominal concentrations. However, this is not acceptable for regulatory submissions by the 2018 US FDA BMV Guidance [19]. Actual QC concentrations based on the amount of calibrator spiked into matrix or buffer should be documented, if possible for the specific biomarker, for DDKs to determine the accuracy of the assay. The kits should also have a minimum of the QCs along the standard curve range and include at least one QC of the endogenous analyte (endogenous QC) in the matrix evaluated for the kit (e.g., human serum). The concentration of the endogenous QC can be determined by assay results tested over 3 days to allow determination of relative accuracy. Alternatively, providing a high concentration stock of the analyte (if stability allows) with sufficient volume from which the end user can prepare QCs would allow users flexibility to prepare QCs in different normal and disease-state matrices and at the appropriate concentrations as needed. Monitoring of both QC types during the validation by the bioanalytical lab informs on the proper strategy for the continued use of the biomarker assay.

\section{Conclusion}

Commercial LBA kits play an important role in regulated bioanalysis. Most CROs, as evidenced by the survey results, like the convenience of using commercial kits. However, as the regulatory scrutiny on biomarker method validation intensifies, bioanalysts working to adapt commercial kits in a regulated environment find themselves in a conundrum. Some kit vendors are aware of the situation and have responded by creating their tiered brands based on the quality and the characterization of the kit. While it is well intentioned, it only adds to the confusion due to a lack of a harmonized approach. The GCC believes that this White Paper will provide a valuable context and clarification around the needs and expectations of the regulated global bioanalysis community. The GCC hopes that this document will provide inputs to generate a harmonized approach to DDKs, which will ultimately help generate quality data for regulatory decision making. 


\section{Future perspective}

The GCC as a global organization will continue to provide recommendations on hot topics of global interest in small- and large-molecule bioanalysis, biomarkers and immunogenicity. Additionally, CRO-Pharma scientific interchange meetings will continue in order to facilitate communication between the two. Please contact the GCC [40] for the exact date and time of future meetings, and for all membership information.

\section{Acknowledgements}

The GCC would like to thank R Islam and his team (Celerion) for their contribution in structuring this White Paper based on the discussions and consensus of the 12th GCC Closed Forum; all the GCC members who took part in the survey, participated and contributed to the discussions on this important topic at the 12th GCC Closed Forum; N Savoie (GCC) for editing the first draft of this document; W Garofolo (GCC) for organizing the logistics of the 12th GCC Closed Forum and coordinating the review of this document.

\section{Financial \& competing interests disclosure}

The authors have no relevant affiliations or financial involvement with any organization or entity with a financial interest in or financial conflict with the subject matter or materials discussed in the manuscript. This includes employment, consultancies, honoraria, stock ownership or options, expert testimony, grants or patents received or pending, or royalties.

No writing assistance was utilized in the production of this manuscript.

\section{References}

1. Premkumar N, Lowes S, Jersey J et al. Formation of a global contract research organization council for bioanalysis. Bioanalysis 2(11), 1797-1800 (2010).

2. Breda N, Garofolo F, Cruz Caturla M et al. The 3rd Global CRO Council for Bioanalysis at the International Reid Bioanalytical Forum. Bioanalysis 3(24), 2721-2727 (2011).

3. Lowes S, Jersey J, Shoup R et al. 4th Global CRO council for Bioanalysis: coadministered drugs stability, EMA/US FDA guidelines, 483s and carryover. Bioanalysis 4(7), 763-768 (2012).

4. Nicholson R, Lowes S, Cruz Caturla M et al. 6th GCC focus on LBA: critical reagents, positive controls and reference standards; specificity for endogenous compounds; biomarkers; biosimilars. Bioanalysis 4(19), 2335-2342 (2012).

5. Rocci M, Lowes S, Shoup R et al. 7th GCC: use of incurred samples; fit-for-purpose validation; solution stability; electronic lab notebooks; hyperlipidemic matrix testing. Bioanalysis 6(20), 2713-2720 (2014).

6. Bower J, Fast D, Garofolo F et al. 8th GCC: consolidated feedback to US FDA on the 2013 draft FDA guidance on bioanalytical method validation. Bioanalysis 6(22), 2957-2963 (2014).

7. Hayes R, LeLacheur R, Dumont I et al. 9th GCC closed forum: CAPA in regulated bioanalysis; method robustness, biosimilars, preclinical method validation, endogenous biomarkers, whole blood stability, regulatory audit experiences and electronic laboratory note books. Bioanalysis 8(6), 487-495 (2016).

8. Cape S, Islam R, Nehls C et al. The 10th GCC Closed Forum: rejected data, GCP in bioanalysis, extract stability, BAV, processed batch acceptance, matrix stability, critical reagents, ELN and data integrity and counteracting fraud. Bioanalysis 9(7), 505-516 (2017).

9. Islam R, Briscoe C, Bower J et al. The 11th GCC Closed Forum: cumulative stability; matrix stability; immunogenicity assays; laboratory manuals; biosimilars; chiral methods; hybrid LBA/LCMS assays; fit-for-purpose validation; China Food and Drug Administration bioanalytical method validation. Bioanalysis 10(7), 433-444 (2018).

10. Lowes S, Jersey J, Shoup R et al. Recommendations on: internal standard criteria, stability, incurred sample reanalysis and recent 483 s by the Global CRO Council for Bioanalysis. Bioanalysis 3(12), 1323-1332 (2011).

11. Boterman $\mathrm{M}$, Doig M, Breda $\mathrm{M}$ et al. Recommendations on the interpretation of the new EMA guideline on bioanalytical method validation by Global CRO Council for Bioanalysis (GCC). Bioanalysis 4(6), 651-660 (2012).

12. Sangster T, Maltas J, Struwe P et al. Recommendations on ISR in multi-analyte assays, QA/bioanalytical consultants and GCP by Global CRO Council for Bioanalysis (GCC). Bioanalysis 4(14), 1723-1730 (2012).

13. Lowes $\mathrm{S}$, Boterman M, Doig M et al. Recommendations on bioanalytical method stability implications of co-administered and coformulated drugs by Global CRO Council for Bioanalysis (GCC). Bioanalysis 4(17), 2117-2126 (2012).

14. Hougton R, Gouty D, Allinson J et al. Recommendations on biomarker bioanalytical method validation by GCC. Bioanalysis 4(20), 2439-2446 (2012).

15. Lowes S, LeLacheur R, Shoup R et al. Recommendations on incurred sample stability (ISS) by GCC. Bioanalysis 6(18), 2385-2390 (2014).

16. Stevenson L, Richards S, Pillutla R et al. 2018 White Paper in bioanalysis part 3: focus on flow cytometry, gene therapy, cut points and key clarifications on BAV. Bioanalysis 10(24), 1973-2001 (2018). 
17. Bowsher RR, Nowatzke W, Sailstad JM, Khan MU. Application of commercial research-grade biomarker assays in drug development: is it time to create "pharmaceutical-grade" kits? Bioanalysis 4(20), 2427-2430 (2012).

18. Islam R, Kar S, Islam C, Farmen R. Adaptation of commercial biomarker kits and proposal for "drug development kits" to support bioanalysis: call for action. Bioanalysis 10(12), 945-955 (2018).

19. US Department of Health and Human Services, US FDA, Center for Drug Evaluation and Research, Center for Veterinary Medicine. Guidance for Industry, Bioanalytical Method Validation. MD, USA (2018).

20. Piccoli SP, Sauer JM, Amur S et al. Points to consider document: scientific and regulatory considerations for the analytical validation of assays used in the qualification of biomarkers in biological matrices.

https://healthpolicy.duke.edu/sites/default/files/atoms/files/cpath_ptc_biomarker_qualification_assays_20170623.pdf

21. Piccoli SP, Garofolo F. Biomarker assay validation. Bioanalysis 10(12), 889-891 (2018).

22. Jani $\mathrm{D}$, Allison J, Berisha $\mathrm{F}$ et al. Recommendations for use and fit-for-purpose validation of biomarker multiplex ligand binding assays in drug development. AAPS J. 18(1), 1-14 (2015).

23. Lee JW, Devanarayan V, Barrett YC et al. Fit-for-purpose method development and validation for successful biomarker measurement. Pharm. Res. 23(2), 312-328 (2006).

24. Amaravadi L, Song A, Myler H et al. 2015 White Paper on recent issues in bioanalysis: focus on new technologies and biomarkers. Bioanalysis 7(24), 3107-3124 (2015).

25. Richards S, Amaravadi L, Pillutla R et al. 2016 White Paper on recent issues in bioanalysis: focus on biomarker assay validation (BAV). Bioanalysis 8(23), 2475-2496 (2016).

26. Gupta S, Richards S, Amaravadi L et al. 2017 White Paper on recent issues in bioanalysis: a global perspective on immunogenicity guidelines and biomarker assay performance. Bioanalysis 9(24), 1967-1996 (2017).

27. Khan MU, Bowsher RR, Cameron $M$ et al. Recommendations for adaptation and validation of commercial kits for biomarker quantification in drug development. Bioanalysis 7(2), 229-242 (2015).

28. Arnold ME, Booth B, King L et al. Workshop Report: crystal city VI-bioanalytical method validation for biomarkers. AAPS J. 18(6), 1366-1372 (2016).

29. FDA-NIH Biomarker Working Group. BEST (Biomarkers, EndpointS, and other Tools) Resource. Silver Spring (MD), Food and Drug Administration (US); Bethesda (MD), National Institutes of Health (USA) (2016).

30. Cowan K, Amaravadi L, Cameron M. Recommendations for selection and characterization of protein biomarker assay calibrator material. AAPS J. 19(6), 1550-1563 (2017).

31. O'Hara DM, Theobald V, Egan AC et al. Ligand binding assays in the 21 st century laboratory: recommendations for characterization and supply of critical reagents. AAPS J. 14(2), 316-328 (2012).

32. Westgard JO, Aronsson T, Groth T, De Verdier CH. Combined Shewhart cusum control chart for improved quality control in clinical chemistry. Clin. Chem. 23(10), 1881-1887 (1977).

33. Ezzelle J, Rodriguez-Chavez IR, Darden JM et al. Guidelines on good clinical laboratory practice: bridging operations between research and clinical research laboratories. J. Pharm. Biomed. Anal. 46(1), 18-29 (2008).

34. King LE, Farley $\mathrm{E}$, Imazato $\mathrm{M}$ et al. Ligand binding assay critical reagents and their stability: recommendations and best practices from the global bioanalysis consortium harmonization team. AAPS J. 16(3), 504-515 (2014).

35. DeSilva B, Garofolo F, Rocci M et al. 2012 White Paper on recent issues in bioanalysis and alignment of multiple guidelines. Bioanalysis 4(18), 2213-2226 (2012).

36. Stevenson L, Rocci M, Garofolo F et al. 2013 White Paper on recent issues in bioanalysis: "hybrid" - the best of LBA \& LC/MS. Bioanalysis 5(23), 2903-2918 (2013).

37. Stevenson LF, Purushothama S. Parallelism: considerations for the development, validation and implementation of PK and biomarker ligand-binding assays. Bioanalysis 6(2), 185-198 (2014).

38. Mehta D, Purushothama S, Stevenson L. Parallelism: the foundation of biomarker assay development and validation. Bioanalysis 10(12), 897-899 (2018).

39. King LE. Parallelism experiments in biomarker ligand-binding assays to assess immunological similarity. Bioanalysis 8(23), 2387-2391 (2016)

40. Global CRO Council for Bioanalysis. www.global-cro-council.org 


\section{Authors}

Rafiq Islam', Sumit Kar ${ }^{1}$, Hanna Ritzén ${ }^{2}$, Amanda Hays ${ }^{3}$, Rabab Tayyem ${ }^{4}$, Colin Barry ${ }^{5}$, Anahita Keyhani ${ }^{6}$, Jennifer Zimmer ${ }^{7}$, Maria Cruz Caturla ${ }^{8}$, Philippe Couerbe ${ }^{9}$, Mark Warren ${ }^{9}$, Ardeshir Khadang $^{10}$, James Bourdage ${ }^{11}$, Kathie Lindley ${ }^{12}$, Dave Williams ${ }^{12}$, Nicola Hughes ${ }^{13}$, Curtis Sheldon ${ }^{1}$, Christina Satterwhite ${ }^{14}$, Jenifer Vija ${ }^{15}$, Mathilde $\mathrm{Yu}^{16}$, Iohann Boulay ${ }^{17}$, John Stamatopoulos ${ }^{17}$, Jenny $\operatorname{Lin}^{18}$, Stephanie Cape ${ }^{19}$, Sian Estdale ${ }^{20}$, Eric Thomas ${ }^{21}$, Andrew Dinan ${ }^{22}$, Robert MacNeill23, Yi Qun Xiao $^{23}$, Wei Garofolo*,24, Natasha Savoie ${ }^{24}$, Michael Brown ${ }^{25}$, Paul Rhyne ${ }^{26}$, George Hristopoulos ${ }^{27}$, Arron $\mathrm{Xu}^{27}$, Lawrence Goodwin ${ }^{28}$, Franklin Spriggs ${ }^{28}$, Allan $\mathrm{Xu}^{29}$, Kayode Awaiye ${ }^{30}$, Roger Hayes ${ }^{31}$, Jessica St Charles ${ }^{31}$, Mohammed Bouhajib32, Chantal DiMarco ${ }^{32}$, Luigi Silvestro33, Simona Rizea Savu ${ }^{33}$, Patrick Bennett ${ }^{34}$, Vellalore Kakkanaiah ${ }^{34}$, Corey Nehls ${ }^{34}$, Bruce Stouffer ${ }^{34}$, Edward Tabler ${ }^{34}$, Chad Briscoe $^{3}$, Shane Karnik ${ }^{35}$, Ira DuBey ${ }^{36}$, Nadia Kulagina ${ }^{36}$, John Lindsay ${ }^{37}$, Chris Beaver ${ }^{38}$, Clark Williard ${ }^{38}$, Hao Wang ${ }^{39}$, Hao Feng ${ }^{40}$, Michele Malone ${ }^{41}$, Edward Wells ${ }^{41}$, Xinping Fang ${ }^{42}$ \& Marc Moussallie ${ }^{42}$

\section{Affiliations}

${ }^{1}$ Celerion, Lincoln, NE, USA

${ }^{2}$ Mercodia AB, Uppsala, Sweden

${ }^{3}$ PRA Health Sciences, Lenexa, KS, USA

${ }^{4}$ ACDIMA Center for Bioequivalence \& Pharmaceutical Studies, Amman, Jordan

${ }^{5}$ Alliance Pharma, Malvern, PA, USA

${ }^{6}$ Altasciences, Laval, QC, Canada

${ }^{7}$ Alturas Analytics, Moscow, ID, USA

${ }^{8}$ Anapharm Bioanalytics, Barcelona, Spain

${ }^{9}$ Atlanbio, Saint-Nazaire, France

${ }^{10}$ Axis Clinicals, Dilworth, MN, USA

${ }^{11}$ BASi, West Lafayette, IN, USA

${ }^{12}$ BioAgilytix, Durham, NC, USA

${ }^{13}$ BioPharma Services, Toronto, ON, Canada

${ }^{14}$ Charles River Laboratories, Reno, NV, USA

${ }^{15}$ Charles River Laboratories, Skokie, IL, USA

${ }^{16}$ CIRION Biopharma Research, Laval, QC, Canada

${ }^{17}$ CiToxLAB North America, Laval, QC, Canada

${ }^{18} \mathrm{CMIC}$, Hoffman Estates, IL, USA

${ }^{19}$ Covance, Madison, WI, USA

${ }^{20}$ Covance, Harrogate, UK

${ }^{21}$ Covance, Indianapolis, IN, USA
${ }^{22}$ CPR Pharma Services, Adelaide, Australia

${ }^{23}$ Envigo, Somerset, NJ, USA

${ }^{24} \mathrm{GCC}$, Toronto, ON, Canada

${ }^{25}$ ICON Laboratory Services, Whitesboro, NY, USA

${ }^{26}$ Immunologix Laboratories, Tampa, FL, USA

${ }^{27}$ Intertek, San Diego, CA, USA

${ }^{28}$ KCAS Bioanalytical \& Biomarker Services, Shawnee Mission, KS, USA

${ }^{29}$ Keystone Bioanalytical, North Wales, PA, USA

${ }^{30}$ Lambda Therapeutic Research, Toronto, ON, Canada

${ }^{31}$ MPI Research, Mattawan, MI, USA

${ }^{32}$ Pharma Medica Research Inc., Mississauga, ON, Canada

${ }^{33}$ Pharma Serv International, Bucharest, Romania

${ }^{34}$ PPD Laboratories, Richmond, VA, USA

${ }^{35}$ Pyxant Labs, Colorado Springs, CO, USA

${ }^{36}$ Smithers Avanza, Gaithersburg, MD, USA

${ }^{37}$ SNBL USA, Everett, WA, USA

${ }^{38}$ Syneos Health, Princeton, NJ, USA

${ }^{39}$ TETRAQ, Herston, Australia

${ }^{40}$ Value Pharmaceutical Services, Nanjing, PR China

${ }^{41}$ Worldwide Clinical Trials, Austin, TX, USA

${ }^{42}$ WuXi Apptec, Plainsboro, NJ, USA 
